\title{
Lateral Pelvic Lymph Node Metastases in Rectal Cancer: A Systematic Review
}

\author{
Y. Atef ${ }^{1} \cdot$ T. W. Koedam ${ }^{1}$ S. E. van Oostendorp ${ }^{1} \cdot$ H. J. Bonjer ${ }^{1}$ A. R. Wijsmuller ${ }^{1} \cdot$ \\ J. B. Tuynman ${ }^{1}$
}

Published online: 29 August 2019

(C) The Author(s) 2019

\begin{abstract}
Background Synchronous lateral pelvic lymph node (LPLN) involvement occurs in a significant number of patients with rectal cancer. The aim of this study is to determine the rate of LPLN metastases in rectal cancer patients with LPLN suspicious for metastases (LPLNSM) on pretreatment imaging, treated with neoadjuvant chemoradiotherapy (nCRT). Additionally, the influence of LPLN responsiveness to nCRT as determined by post-nCRT restaging scan was investigated.

Methods A systematic review was conducted to identify studies on patients with author-defined LPLNSM that reported the pathological outcomes after total mesorectal excision (TME) with lateral pelvic lymph node dissection (LPLD). MEDLINE, EMBASE, Web of Science and the Cochrane Library were searched. The primary outcome was the percentage of pathologically confirmed LPLN metastases.

Results A total of 462 patients from eleven studies were identified. The number of pathologically confirmed LPLN metastases in 361 patients that underwent uni- or bilateral LPLD ranged from 21.9 to $61.1 \%$. The LPLD resulted in pathologically confirmed metastases in a range from 0 to $20.4 \%$ of patients with responsive LPLNSM and in a range from 25.0 to $83.3 \%$ of patients with persistent nodes. However, radiologic cutoff criteria for the evaluation of LPLN differed between studies.

Conclusions In a large number of patients with LPLNSM on initial imaging, metastatic LPLN are present after nCRT and surgical treatment. Even in LPLN that are considered responsive on restaging, significant rates of pathologically confirmed metastases are reported.
\end{abstract}

\section{Introduction}

Lateral pelvic lymph node (LPLN) metastases are present in $14-20 \%$ of all rectal cancer cases [1-6]. According to some studies, there is LPLN involvement in the majority of

Electronic supplementary material The online version of this article (https://doi.org/10.1007/s00268-019-05135-3) contains supplementary material, which is available to authorized users.

\section{A. R. Wijsmuller} a.r.wijsmuller@umcg.nl

1 Department of Surgery, VU University Medical Center, Amsterdam, The Netherlands patients with a locoregional recurrence, while other studies emphasize the role of residual mesorectal fat with cancer cells or the omission of a rectal washout as cause of recurrence [3-5, 7-9]. The LPLN compartment includes the common, internal and external iliac and the obturator artery lymph nodes [10].

Different treatment strategies have been adopted worldwide for rectal cancer patients with LPLN suspicious for metastases (LPLNSM), and optimal management remains controversial. According to European and American guidelines, standard treatment consists of neoadjuvant chemoradiotherapy (nCRT) followed by a total mesorectal excision (TME) [11-14]. By most of these guidelines, the 
possibility of a (selective) lateral pelvic lymph node dissection (LPLD) is mentioned without discussing its necessity in case of LPLNSM that are persistent or responsive after nCRT [12-14]. The French guidelines for the treatment of rectal cancer advise against LPLD and recommend a lymph node excision in case of LPLNSM and positioning of a fiducial marker to help further irradiation targeting [11]. Hesitation to perform LPLD might be based on a presumed increase in morbidity. However, in the East, removal of LPLN is considered an important extension, since its addition to an oncologic mesorectal excision in primary low/mid rectal cancer patients without LPLNSM was reported to reduce the local recurrence rate [15]. The Japanese Society for Cancer of the Colon and Rectum (JSCCR) guidelines recommend TME with LPLD as standard treatment for patients whose lower tumor border is located distal to the peritoneal reflection and whose tumor has invaded beyond the muscularis propria [16]. For this subgroup of patients without nCRT, LPLN metastases were reported in $20 \%$ [16].

Differences exist between Western and Eastern approach to rectal carcinoma with LPLNSM. Whether a LPLD should be performed during TME depends on the risk of metastases after nCRT. The aim of this review study is to determine the percentage of LPLN metastases in patients with rectal carcinoma and LPLNSM on pretreatment imaging, treated with nCRT.

\section{Materials and methods}

Studies that reported the pathological outcomes after TME with LPLD after nCRT for LPLNSM on pretreatment imaging were reviewed. The search strategy is detailed in supplementary Table 1 in the appendix. Randomized, prospective and retrospective cohort studies were eligible for inclusion. The electronic databases MEDLINE, EMBASE, Web of Science and Cochrane library were searched from inception to the 17th of May 2018. Reference lists of the included studies were cross-checked for additional papers. Studies identified by the searches were sifted based on title, abstract and full text by a single reviewer (Fig. 1). Two reviewers (YA, ARW) assessed the full texts of the remaining studies independently. This review was conducted in accordance with PRISMA guidelines [17]. The quality of evidence was rated by means of GRADE methodology for the primary outcome [18, 19]. An evidence table and summary of findings table was produced. Authors were contacted by electronic mail in the event of any doubt. This review has been registered on PROSPERO international register of systematic reviews with registration number: CRD42018091327.
The primary outcome was the percentage of pathologically confirmed LPLN metastases in rectal cancer patients with LPLNSM on initial imaging, treated with nCRT. Data extracted included author, year of publication, type of study, number of patients, number of LPLD in case the number of patients was not reported, type of imaging modality, author-defined cutoff criteria for LPLNSM before and after nCRT, nCRT regimen (Supplementary Table 2), interval between nCRT and surgery (Supplementary Table 2), percentage of persistent/responsive nodes after nCRT and percentage of patients/LPLD with pathologically confirmed LPLN metastases for both persistent and responsive LPLNSM.

\section{Results}

The literature search (Supplementary Table 1) identified 5892 initial articles, of which 1112 duplicates were removed, and 4696 articles were excluded on the basis of title or abstract (Fig. 1). Eleven studies fulfilled all of the selection criteria after full-text review of the remaining 84 articles [20-30]. These included two prospective [20, 21] and nine retrospective cohort studies [22-30]. The quality of evidence of the four comparative [26-28, 30] and seven non-comparative cohort studies [20-25, 29] was low because of the observational nature. However, the level of evidence was upgraded to moderate, since the chance at bias with a dichotomous outcome measure, as the one used in our study, is little. Ranging from eight to 77 patients per study, a total of 462 patients were included. An overview of the type of studies, number of patients, their pathology outcome and imaging modalities can be found in Table 1 . Cutoff criteria and node aspects for the assessment of LPLNSM before and after nCRT were reported by the majority of studies (Table 2, 3). Radiologic cutoff criteria for the evaluation of LPLN differed between studies as noted in the tables (Table 2, 3). The LPLN pathology outcomes of responsive and persistent LPLNSM after nCRT are displayed in Tables 4 and 5, respectively. Two of the eleven included studies described their results on pathological outcome of the LPLN based on the number of excisions [20, 21], meaning that bilateral dissection was counted as two excisions. The rest of the studies described the pathological outcome of the LPLN based on the number of patients. Pathologically confirmed metastases occurred in a range from 39.0 to $71.1 \%$ of all LPLD [20, 21]. In the other nine studies, pathologically confirmed LPLN occurred in a range from 25.0 to $61.1 \%$ of all patients had after uni- or bilateral LPLD. Only five $[22,23,26,27,29]$ and seven studies [22-27, 29] provided information about patients with responsive and persistent nodes, respectively. Patients with responsive LPLNSM 
Fig. 1 Study flow diagram

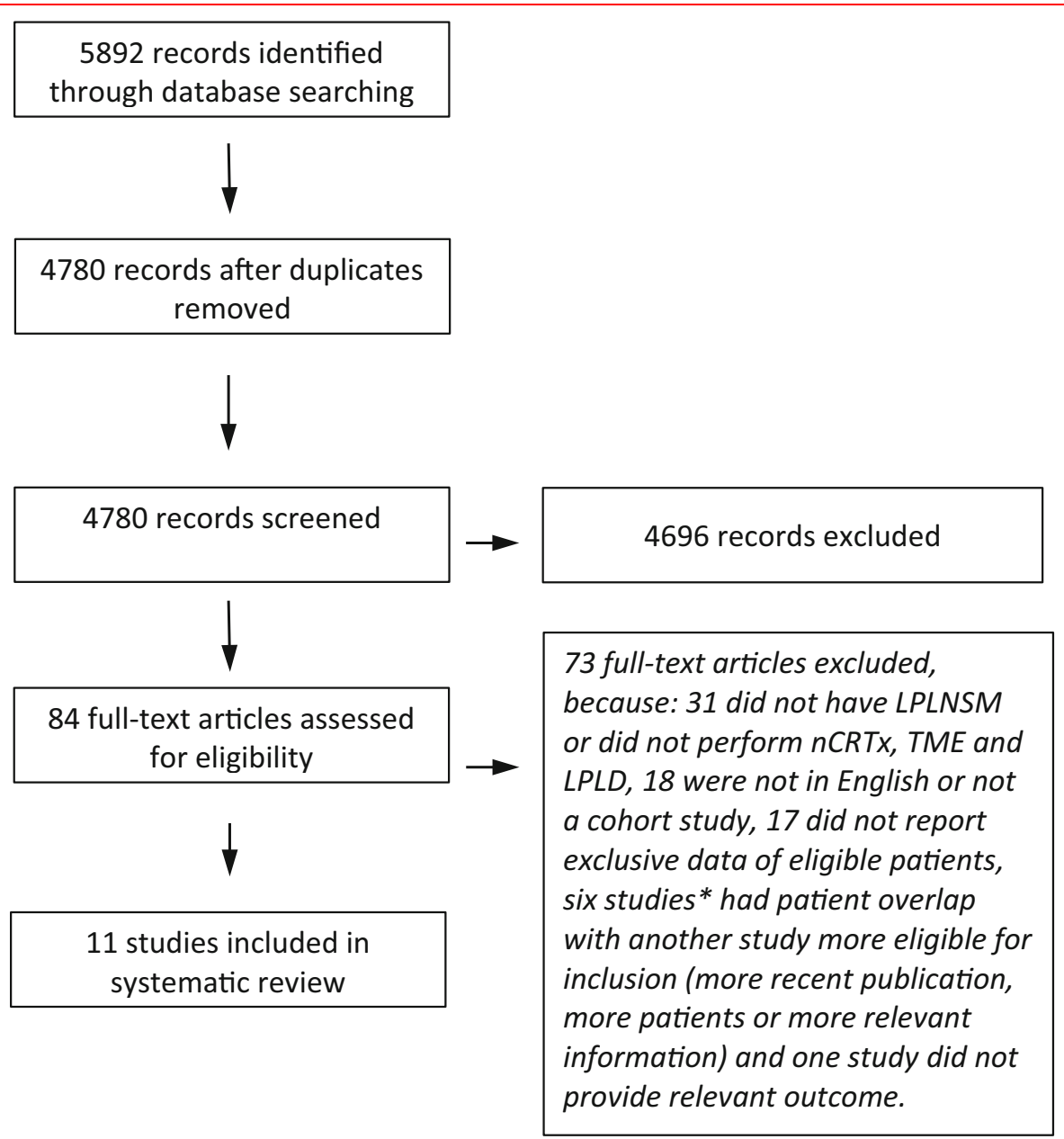

* Akiyoshi et al. $2013^{53}$ was excluded due to patient overlap with Akiyoshi et al. $2015^{23}$

* Nagasaki et al. $2017^{54}$ was excluded due to patient overlap with Akiyoshi et al. $2015^{23}$

* Ogura et al. $2017^{55}$ was excluded due to patient overlap with Akiyoshi et al. $2015^{23}$

* Ishihara et al. $2018^{48}$ was excluded due to patient overlap with Ishihara et al. $2017^{26}$

* Otowa et al. $2011^{56}$ was excluded due to patient overlap with Matsuda et al. $2018^{30}$

* Park et al. $2011^{57}$ was excluded due to patient overlap with Kim, M. J. et al. $2017^{27}$

turned out to have LPLN metastases in a range from 0 to 20.4\%. Patients with persistent LPLNSM turned out to have LPLN metastases in a range from 25.0 to $83.3 \%$.

\section{Discussion}

To our knowledge, this is the first review study on pathologically confirmed LPLN metastases in rectal cancer patients with LPLNSM, treated with nCRT. LPLN metastases occurred in a large amount of patients with LPLNSM on initial imaging and patients with persistent nodes after nCRT. Even in LPLN that are considered responsive on restaging, in a significant amount of patients pathologically confirmed metastases are reported. These data suggest that
LPLD seems to be justified in case of LPLNSM that are non-responsive to nCRT and should even be considered in LPLN that are responsive. This study should alert surgeons that are currently executing diverging policies.

The main limitation of retrospective studies investigating long-term outcomes for treatment of patients with LPLNSM is a lack of histological verification of the nature of LPLN. Brown et al. retrospectively reviewed baseline MRI images of rectal cancer patients that underwent a TME or abdominoperineal resection with or without neoadjuvant treatment for a minimum of 5-year follow-up [31]. A high suspicion of LPLNSM was issued in case of the presence of mixed signal intensity and/or an irregular border of the nodal capsule, irrespective of nodal size. MRI detected LPLNSM was neither biopsied nor evaluated by 
Table 1 Characteristics of studies and pathological results of LPLNSM

\begin{tabular}{|c|c|c|c|c|}
\hline References & Type of study & $\begin{array}{l}\text { Imaging modalities to } \\
\text { identify LPLNSM }\end{array}$ & $\begin{array}{l}\text { Total amount } \\
\text { of patients, } N\end{array}$ & $\begin{array}{l}\text { PA confirmed } \\
\text { LPLN after LPLD }\end{array}$ \\
\hline Liang [20] & Prospective & MRI, CT, PET, TRUS & 34 (45 excisions) $)^{\mathrm{b}, \mathrm{c}}$ & $32(71.1)^{\mathrm{b}}$ \\
\hline Lim et al. [21] & Prospective & MRI, TRUS & 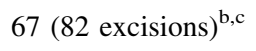 & $32(39.0)^{\mathrm{b}}$ \\
\hline Oh et al. [22] & Retrospective & MRI & 66 & $22(33.3)$ \\
\hline Akiyoshi et al. [23] & Retrospective & MRI, MDCT & 77 & $31(40.3)$ \\
\hline Sinukumar et al. [24] & Retrospective & MRI & $8^{\mathrm{c}}$ & $2(25.0)$ \\
\hline Shin et al. [25] & Retrospective & MRI, CT & $18^{\mathrm{c}}$ & $11(61.1)$ \\
\hline Ishihara et al. [26] & Retrospective & MRI, CT & 31 & $16(51.6)$ \\
\hline Kim et al. [27] & Retrospective & MRI, CT, PET & 53 & $20(37.7)$ \\
\hline Yamaoka et al. [28] & Retrospective & MRI & $19^{\mathrm{d}}$ & $7(36.8)$ \\
\hline Kim et al. [29] & Retrospective & MRI & 57 & $23(40.4)$ \\
\hline Matsuda et al. [30] & Retrospective & MRI, CT, PET & $32^{\mathrm{a}, \mathrm{d}}$ & $7(21.9)$ \\
\hline Total patients & & & 462 & \\
\hline Total patients ${ }^{\mathrm{e}}$ & & & 361 & $139(21.9-61.1)$ \\
\hline Total excisions & & & $127^{\mathrm{b}}$ & $64(39.0-71.1)^{\mathrm{b}}$ \\
\hline
\end{tabular}

LPLN(SM) lateral pelvic lymph nodes (suspicious for metastases), MRI magnetic resonance imaging, $C T$ computed tomography, $M D C T$ multidetector CT, PET positron emission tomography, TRUS transrectal ultrasonography, $P A$ pathology, $L P L D$ lateral pelvic lymph node dissection

${ }^{\mathrm{a}}$ Correspondence with author

${ }^{\mathrm{b}}$ Studies reporting the number of excisions instead of patients. Information per patients was not reported and could not be identified through communication with author

${ }^{\mathrm{c}}$ Only patients/excisions with persistent LPLNSM after nCRTx were included in the study

${ }^{\mathrm{d}}$ The study did not report any information about the ratio of persistent and responsive nodes after nCRT

${ }^{e}$ Only patients of the studies reporting ouctomes per patients, without the patients from studies that only report the results of the excisions

Table 2 Cutoff criteria for the assessment LPLNSM before nCRTx

\begin{tabular}{llll}
\hline References & \multicolumn{2}{l}{ Cutoff criteria LPLNSM before nCRTx } \\
\cline { 2 - 4 } & Short-axis diameter & Long-axis diameter & Aspect \\
\hline Liang [20] & NR & NR & NR \\
Lim et al. [21] & NR & NR & NR \\
Oh et al. [22] & $>5 \mathrm{~mm}$ & - & NR \\
Akiyoshi et al. [23] & - & $\geq 7 \mathrm{~mm}$ & $\mathrm{NR}$ \\
Sinukumar et al. [24] & $\geq 8 \mathrm{~mm}^{\mathrm{a}}$ & - & $\mathrm{NR}$ \\
Shin et al. [25] & - & $\geq 7 \mathrm{~mm}$ & $\mathrm{NR}$ \\
Ishihara et al. [26] & $\geq 8 \mathrm{~mm}$ & & NR \\
Kim et al. [27] & $\geq 5 \mathrm{~mm}$ & - & Morphologic changes \\
Yamaoka et al. [28] & $>5 \mathrm{~mm}$ & - & NR \\
Kim et al. [29] & $\geq 5 \mathrm{~mm}$ & - & NR uptake on PET/CT \\
Matsuda et al. [30] & $\geq 8 \mathrm{~mm}$ & - & High-intensity spot on PET
\end{tabular}

$L P L N(S M)$ lateral pelvic lymph nodes (suspicious for metastases), $n C R T x$ neoadjuvant chemoradiation therapy, $N R$ not reported

${ }^{\mathrm{a}}$ Correspondence with author: $\geq 8 \mathrm{~mm}$ short-axis diameter or any node suspected by local radiologist

${ }^{\mathrm{b}}$ With spiculated/indistinct borders or mottled heterogenic pattern

LPLD. The authors reported a 5-year disease-free survival of 42 and $71 \%$, respectively, for 38 patients with MRIidentified LPLNSM at baseline and 287 patients without suspicious nodal characteristics. Kusters et al. conducted a study that compared the Dutch and Japanese approach for low rectal cancer: TME with/without neoadjuvant RTx compared to extended surgery consisting of LPLD and an oncologic rectal resection without any (neo) adjuvant treatment in the majority of cases, respectively [32]. The authors reported good local control for both approaches, as 
Table 3 Cutoff criteria for the assessment LPLNSM after nCRTx

\begin{tabular}{|c|c|c|c|}
\hline \multirow[t]{2}{*}{ References } & \multicolumn{3}{|c|}{ Cutoff criteria LPLNSM after nCRTx (persistent nodes) } \\
\hline & Short-axis diameter & Long-axis diameter & Aspect \\
\hline Liang [20] & NR & & \\
\hline Lim et al. [21] & $\geq 5 \mathrm{~mm}^{\mathrm{b}}$ & - & Morphologic changes ${ }^{\mathrm{b}}$ \\
\hline Oh et al. [22] & $>5 \mathrm{~mm}$ & - & NR \\
\hline Akiyoshi et al. [23] & $>5 \mathrm{~mm}$ & - & NR \\
\hline Sinukumar et al. [24] & $\geq 8 \mathrm{~mm}^{\mathrm{a}}$ & - & NR \\
\hline Shin et al. [25] & - & $\geq 4 \mathrm{~mm}$ & NR \\
\hline Ishihara et al. [26] & $\geq 8 \mathrm{~mm}$ & - & NR \\
\hline Kim et al. [27] & $\geq 5 \mathrm{~mm}$ & - & - \\
\hline Yamaoka et al. [28] & - & - & NR \\
\hline Kim et al. [29] & $>5 \mathrm{~mm}$ & - & - \\
\hline Matsuda et al. [30] & NR & NR & NR \\
\hline
\end{tabular}

$L P L N(S M)$ lateral pelvic lymph nodes (suspicious for metastases), $n C R T x$ neoadjuvant chemoradiation therapy, $N R$ not reported

${ }^{a}$ Correspondence with author: $\geq 8 \mathrm{~mm}$ short-axis diameter or any node suspected by local radiologist

${ }^{b} \geq 5 \mathrm{~mm}$ in the largest short-axis diameter and/or a spiculated or indistinct border and/or a mottled heterogenic pattern

Table 4 Results of patients with responsive LPLNSM after nCRTx

\begin{tabular}{llll}
\hline References & $\begin{array}{l}\text { Total amount of patients, } \\
N\end{array}$ & $\begin{array}{l}\text { Patients with responsive LPLNSM } \\
\text { after nCRTx, } N(\%)\end{array}$ & $\begin{array}{l}\text { PA confirmed LPLN } \\
\text { after LPLD, } N(\%)\end{array}$ \\
\hline Oh et al. [22] & 66 & $30(45.5)$ & 0 \\
Akiyoshi et al. [23] & 77 & $49(63.6)$ & $10(20.4)$ \\
Ishihara et al. [26] & 31 & $11(35.5)$ & $1(9.1)$ \\
Kim et al. [27] & 53 & $30(56.6)$ & $5(16.7)$ \\
Kim et al. [29] & 57 & $33(57.9)$ & $3(9.1)$ \\
Total patients & 284 & $153(35.5-63.6)$ & $16(0-20.4)$
\end{tabular}

$L P L N(S M)$ lateral pelvic lymph nodes (suspicious for metastases), $n C R T x$ neoadjuvant chemoradiotherapy, $P A$ pathology, $L P L D$ lateral pelvic lymph node dissection

compared to TME alone, and therefore, the added value of the application of uni- or bilateral LPLD on the prevention of lateral recurrence was questioned [32]. Kim et al. conducted a retrospective study specifically on long-term outcomes such as local recurrence and overall survival. The authors analyzed 580 patients who underwent nCRT and TME. The results suggest that a subgroup with responsive nodes after nCRT may not benefit from LPLD and a subgroup with persistent LPLNSM after nCRT may benefit from LPLD [33]. The current study did not analyze any long-term outcomes, which is a limitation of this study. However, long-term outcomes rather describe the indirect effect than the direct effect of nCRT for clinically positive LPLN, since other oncological factors might also contribute to recurrence and survival.

Besides oncologic outcomes, the morbidity associated with LPLD should also be taken into account such as urogenital and bowel dysfunction and motor dysfunction of the lower extremity [34-36]. Such morbidity has been reported [36-38]. However, a recent RCT did not show any significant differences in postoperative morbidity by adding LPLD to TME despite a longer operation time and a higher amount of blood loss [39]. Nonetheless, Asian patients were treated in this trial, and therefore, morbidity associated with LPLD might be higher in a European population.

An accurate restaging after nCRT is of paramount importance. Promising techniques to improve the accuracy of nodal (re)staging include diffusion-weighted imaging (DWI), lymph node-specific contrast agents and PET-CT. Van Heeswijk et al. compared clinical rectal lymph node positivity according to DWI after nCRT with the number of metastatic nodes at histopathology or long-term follow-up [40]. They analyzed 90 rectal cancer patients including 71 patients with a yNO status and 19 patients with a yN-positive status. To differentiate between yNO and yN-positive 
Table 5 Results of patients with persistent LPLNSM after nCRTx

\begin{tabular}{llll}
\hline References & $\begin{array}{l}\text { Total amount of } \\
\text { patients, } N\end{array}$ & $\begin{array}{l}\text { Persistent LPLNSM after } \\
\text { nCRTx, } N(\%)\end{array}$ & $\begin{array}{l}\text { PA confirmed LPLN after } \\
\text { LPLD, } N(\%)\end{array}$ \\
\hline Liang [20] & $34(45 \text { excisions })^{\mathrm{a}, \mathrm{b}}$ & $45(100)^{\mathrm{a}}$ & $32(71.1)^{\mathrm{a}}$ \\
Lim et al. [21] & $67(82 \text { excisions })^{\mathrm{a}, \mathrm{b}}$ & $82(100)^{\mathrm{a}}$ & $32(39.0)^{\mathrm{a}}$ \\
Oh et al. [22] & 66 & $36(54.5)$ & $22(61.1)$ \\
Akiyoshi et al. [23] & 77 & $28(36.4)$ & $21(75.0)$ \\
Sinukumar et al. [24] & $8^{\mathrm{b}}$ & $8(100)$ & $2(25.0)$ \\
Shin et al. [25] & $18^{\mathrm{b}}$ & $18(100)$ & $11(61.1)$ \\
Ishihara et al. [26] & 31 & $20(64.5)$ & $15(75.0)$ \\
Kim et al. [27] & 53 & $23(43.4)$ & $15(62.5)$ \\
Kim et al. [29] & 57 & $24(42.1)$ & $20(83.3)$ \\
Total patients & 411 & & $157(36.4-100)$ \\
Total patients & 310 & $127(100)^{\mathrm{a}}$ & $64(39.0-71.1)^{\mathrm{a}}$
\end{tabular}

$L P L N(S M)$ lateral pelvic lymph nodes (suspicious for metastases), $n C R T x$ neoadjuvant chemoradiotherapy, $P A$ pathology, $L P L D$ lateral pelvic lymph node dissection

${ }^{a}$ Studies reporting the number of excisions instead of patients. Information per patients was not reported and could not be identified through communication with author

${ }^{\mathrm{b}}$ Only patients/excisions with persistent LPLNSM after nCRTx were included in the study

${ }^{\mathrm{c} O n l y}$ patients of the studies reporting ouctomes per patients, without the patients from studies that only report the results of the excisions

status, the positive predictive value was $24 \%$ and the negative predictive value was $100 \%$, concluding that absence of nodes at DWI is a reliable predictor of yN0 status after nCRT. An earlier study from the same group demonstrated a high negative predictive value for prediction of nodal status $(0.95$ and 0.85 for expert and general radiologist, respectively) in case of MRI with a lymph node-specific contrast agent [41]. Ishihara et al. evaluated the diagnostic value of PET-CT in 34 patients treated with nCRT and reported a high degree of accuracy for the prediction of metastatic LPLN [42]. Most recent studies suggest nodal size to be one of the most important risk factors for node positivity. Ogura et al. performed a multicentre pooled analysis of patients with low, locally advanced rectal cancer from 12 hospitals in seven Eastern and Western countries [43]. LPLD was performed in 142 patients in five hospitals, which resulted in 35 patients (24.6\%) with pathologically positive LPLN. After multivariable analysis, LPLN with a short axis equal to or greater than $7 \mathrm{~mm}$ resulted in a significantly higher risk of lateral local recurrence (HR 2060; $P=0.045$ ) compared with LPLN of less than $7 \mathrm{~mm}$.

Up till now, to our knowledge, no results of RCT's have been reported yet, investigating the value of LPLD in case of LPLNSM. Wei et al. are currently enrolling patients with LPLNSM in what they state to be the first clinical trial randomizing patients to either nCRT with TME alone or nCRT with TME combined with LPLD [44]. The randomization of patients in this study is executed prior to nCRT and irrespective of restaging, which implies that in the TME alone group there might be patients with persistent nodes that are not dissected. Although this study investigates a policy that currently is being executed, in light of the results of the present study, this study raises ethical concerns, since a significant number of patients with LPLNSM on pretreatment imaging turned out to have metastatic nodes. These nodes would be left untreated by this study.

It must be noted that four of the studies in this review only included patients with persistent LPLNSM after nCRT $[20,21,24,25]$. Therefore, the percentage of LPLN metastases in these studies is expected to be higher than the percentage of LPLN metastases in the other included studies that also performed LPLD on patients with responsive LPLN after nCRT. This is supported by the data of this review showing LPLN metastases in a range from 25.0 to $83.3 \%$ of patients with persistent nodes and in range from 0 to $20.4 \%$ of patients with responsive nodes.

This current study has several limitations. First of all, variation in the use of imaging modalities to diagnose LPLNSM has possibly contributed to the interpretation of the measured values. A study of the JSCCR mentions that using MRI for identifying the cutoff value is presumably the best imaging modality, due to its simplicity and good interobserver agreement [45]. The use of other imaging modalities like transrectal ultrasound in the studies that were included in this systematic review could have negatively influenced the comparability. Secondly, cutoff criteria and node aspects for determining LPLNSM on imaging modalities were not reported by all studies and 
different cutoff values were used (Table 2, 3, 4, 5). The current review included studies with different cutoff values for lymph nodes to be considered 'suspicious'. Therefore, staging accuracy of the majority of studies seems suboptimal and a selection bias cannot totally be excluded. Thirdly, the amount of time in between nCRT completion and surgery, the dose of radiation therapy and the type of CRT regimen could be of influence on the extent of downstaging and pathologic complete response (pCR) rate by analogy with the response of the primary rectal tumor itself. RCTs support surgical resection of the primary rectal tumor more than 6-8 weeks after nCRT due to more downstaging and a higher $\mathrm{pCR}$ rate, although a survival benefit is unproven [46]. Additionally, results from studies investigating the dose of radiation therapy and the influence of a neoadjuvant regimen incorporating induction chemotherapy on the responsiveness of LPLNSM are of interest.

\section{Conclusions}

In conclusion, in a large amount of patients with LPLNSM on initial imaging, the LPLD pathology report indicates lymphatic metastases after nCRT. Even LPLNSM that are responsive on restaging scans show significant rates of pathologically confirmed metastases. The wide range of pathologically confirmed LPLN metastases illustrates the heterogeneity among studies with a worrisome lower limit. Based on these findings, in patients with LPLNSM on pretreatment imaging, the potential for LPLN metastases after nCRT must be emphasized. Although the review is limited by small, mostly retrospective series and varying methods of LPLN staging, the reported high rates of pathologically confirmed LPLN metastases, a dichotomous outcome measure with a low chance at bias by itself, are worrisome. In the present era with increasing centralization of rectal cancer care, the complexity of LPLD does not seem an impediment.

Open Access This article is distributed under the terms of the Creative Commons Attribution 4.0 International License (http://crea tivecommons.org/licenses/by/4.0/), which permits unrestricted use, distribution, and reproduction in any medium, provided you give appropriate credit to the original author(s) and the source, provide a link to the Creative Commons license, and indicate if changes were made.

\section{References}

1. Yano H, Moran BJ (2008) The incidence of lateral pelvic sidewall nodal involvement in low rectal cancer may be similar in Japan and the West. Br J Surg 95(1):33-49
2. Sato H, Maeda K, Maruta M (2011) Prognostic significance of lateral lymph node dissection in node positive low rectal carcinoma. Int J Colorectal Dis 26(7):881-889

3. Kim TG, Park W, Choi DH, Park HC, Kim SH, Cho YB, Yun SH, Kim HC, Lee WY, Lee J, Park JO, Park YS, Lim HY, Kang WK, Chun HK (2014) Factors associated with lateral pelvic recurrence after curative resection following neoadjuvant chemoradiotherapy in rectal cancer patients. Int J Colorectal Dis 29(2):193-200

4. Kim TH, Jeong SY, Choi DH, Kim DY, Jung KH, Moon SH, Chang HJ, Lim SB, Choi HS, Park JG (2008) Lateral lymph node metastasis is a major cause of locoregional recurrence in rectal cancer treated with preoperative chemoradiotherapy and curative resection. Ann Surg Oncol 15(3):729-737

5. Kim MJ, Kim DY, Kim SY, Baek JY, Chang HJ, Park SC, Park JW, Oh JH (2015) Can chemoradiation allow for omission of lateral pelvic node dissection for locally advanced rectal cancer? J Surg Oncol 111(4):459-464

6. Sugihara K, Kobayashi H, Kato T, Mori T, Mochizuki H, Kameoka S, Shirouzu K, Muto T (2006) Indication and benefit of pelvic sidewall dissection for rectal cancer. Dis Colon Rectum 49(11):1663-1672

7. Fujita S, Mizusawa J, Kanemitsu Y, Ito M, Kinugasa Y, Komori K, Ohue M, Ota M, Akazai Y, Shiozawa M, Yamaguchi T, Bandou H, Katsumata K, Murata K, Akagi Y, Takiguchi N, Saida Y, Nakamura K, Fukuda H, Akasu T, Moriya Y, Colorectal Cancer Study Group of Japan Clinical Oncology G (2017) Mesorectal excision with or without lateral lymph node dissection for clinical stage II/III lower rectal cancer (JCOG0212): a multicenter, randomized controlled, noninferiority trial. Ann Surg 266(2):201-207

8. Syk E, Torkzad MR, Blomqvist L, Ljungqvist O, Glimelius B (2006) Radiological findings do not support lateral residual tumour as a major cause of local recurrence of rectal cancer. Br J Surg 93(1):113-119

9. Kodeda K, Holmberg E, Jorgren F, Nordgren S, Lindmark G (2010) Rectal washout and local recurrence of cancer after anterior resection. Br J Surg 97(10):1589-1597

10. Nakamura T, Watanabe M (2013) Lateral lymph node dissection for lower rectal cancer. World J Surg 37(8):1808-1813

11. Gerard JP, Andre T, Bibeau F, Conroy T, Legoux JL, Portier G, Bosset JF, Cadiot G, Bouche O, Bedenne L, Societe Francaise de Chirurgie Digestive SFdEDSFdRO (2017) Rectal cancer: French Intergroup clinical practice guidelines for diagnosis, treatments and follow-up (SNFGE, FFCD, GERCOR, UNICANCER, SFCD, SFED, SFRO). Dig Liver Dis 49(4):359-367

12. Glynne-Jones R, Wyrwicz L, Tiret E, Brown G, Rodel C, Cervantes A, Arnold D, Committee EG (2017) Rectal cancer: ESMO Clinical Practice Guidelines for diagnosis, treatment and followup. Ann Oncol. 28(suppl_4):iv22-iv40

13. Tumoren LwGI (2017) Landelijke richtlijn: Primaire behandeling rectumcarcinoom

14. Benson AB 3rd, Bekaii-Saab T, Chan E, Chen YJ, Choti MA, Cooper HS, Engstrom PF, Enzinger PC, Fakih MG, Fuchs CS, Grem JL, Hunt S, Leong LA, Lin E, Martin MG, May KS, Mulcahy MF, Murphy K, Rohren E, Ryan DP, Saltz L, Sharma S, Shibata D, Skibber JM, Small W Jr, Sofocleous CT, Venook AP, Willett CG, Freedman-Cass DA, Gregory KM (2012) Rectal cancer. J Natl Compr Cancer Netw 10(12):1528-1564

15. Suzuki K, Muto T, Sawada T (1995) Prevention of local recurrence by extended lymphadenectomy for rectal cancer. Surg Today 25(9):795-801

16. Watanabe T, Muro K, Ajioka Y, Hashiguchi Y, Ito Y, Saito Y, Hamaguchi $T$, Ishida $H$, Ishiguro M, Ishihara S, Kanemitsu $Y$, Kawano H, Kinugasa Y, Kokudo N, Murofushi K, Nakajima T, Oka S, Sakai Y, Tsuji A, Uehara K, Ueno H, Yamazaki K, Yoshida M, Yoshino T, Boku N, Fujimori T, Itabashi M, 
Koinuma N, Morita T, Nishimura G, Sakata Y, Shimada Y, Takahashi K, Tanaka S, Tsuruta O, Yamaguchi T, Yamaguchi N, Tanaka T, Kotake K, Sugihara K, Japanese Society for Cancer of the C, Rectum (2017) Japanese Society for Cancer of the Colon and Rectum (JSCCR) guidelines 2016 for the treatment of colorectal cancer. Int J Clin Oncol

17. Moher D, Shamseer L, Clarke M, Ghersi D, Liberati A, Petticrew M, Shekelle P, Stewart LA, Group P-P (2015) Preferred reporting items for systematic review and meta-analysis protocols (PRISMA-P) 2015 statement. Syst Rev. 4:1

18. GRADE Handbook (2013) GRADE [Handbook]. https://gdt.gra depro.org/app/handbook/handbook.html\#h.3183vuv3ey12

19. Howard Balshem MH, Holger J. Schünemann, Andrew D. Oxman, Regina Kunz, Jan Brozek, Gunn E. Vist, Yngve FalckYtter, Joerg Meerpohl, Susan Norris, Gordon H. Guyatt (2011) GRADE guidelines: 3 . Rating the quality of evidence. J Clin Epidemiol 65(4):401-406

20. Liang JT (2011) Technical feasibility of laparoscopic lateral pelvic lymph node dissection for patients with low rectal cancer after concurrent chemoradiation therapy. Ann Surg Oncol 18(1):153-159

21. Lim SB, Yu CS, Kim CW, Yoon YS, Park SH, Kim TW, Kim JH, Kim JC (2013) Clinical implication of additional selective lateral lymph node excision in patients with locally advanced rectal cancer who underwent preoperative chemoradiotherapy. Int $\mathbf{J}$ Colorectal Dis 28(12):1667-1674

22. Oh HK, Kang SB, Lee SM, Lee SY, Ihn MH, Kim DW, Park JH, Kim YH, Lee KH, Kim JS, Kim JW, Kim JH, Chang TY, Park SC, Sohn DK, Oh JH, Park JW, Ryoo SB, Jeong SY, Park KJ (2014) Neoadjuvant chemoradiotherapy affects the indications for lateral pelvic node dissection in mid/low rectal cancer with clinically suspected lateral node involvement: a multicenter retrospective cohort study. Ann Surg Oncol 21(7):2280-2287

23. Akiyoshi $T$, Matsueda $K$, Hiratsuka M, Unno T, Nagata J, Nagasaki T, Konishi T, Fujimoto Y, Nagayama S, Fukunaga Y, Ueno M (2015) Indications for lateral pelvic lymph node dissection based on magnetic resonance imaging before and after preoperative chemoradiotherapy in patients with advanced lowrectal cancer. Ann Surg Oncol 22(Suppl 3):S614-620

24. Sinukumar S, Engineer R, Saklani A (2015) Preliminary experience with lateral pelvic lymph node dissection in locally advanced rectal cancer. Indian J Gastroenterol 34(4):320-324

25. Ui Sup Shin YNY, Alexander T. Nguyen, Brian K. Bednarski, Craig Messick, Dipen M. Maru, Erin M. Dean, Sa T. Nguyen, Chung-Yuan Hu, George J. Chang. Oncologic Outcomes of Extended Robotic Resection for Rectal Cancer. Annals of Surgical Oncology. 2016;23(7):2249-2257

26. Ishihara S, Kawai K, Tanaka T, Kiyomatsu T, Hata K, Nozawa H, Morikawa T, Watanabe T (2017) Oncological outcomes of lateral pelvic lymph node metastasis in rectal cancer treated with preoperative chemoradiotherapy. Dis Colon Rectum 60(5):469-476

27. Kim HJ, Choi GS, Park JS, Park SY, Cho SH, Lee SJ, Kang BW, Kim JG (2017) Optimal treatment strategies for clinically suspicious lateral pelvic lymph node metastasis in rectal cancer. Oncotarget 8(59):100724-100733

28. Yamaoka Y, Kinugasa Y, Shiomi A, Yamaguchi T, Kagawa H, Yamakawa Y, Numata M, Furutani A (2017) Preoperative chemoradiotherapy changes the size criterion for predicting lateral lymph node metastasis in lower rectal cancer. Int J Colorectal Dis 32(11): $1631-1637$

29. Kim MJ, Hur BY, Lee ES, Parka B, Joo J, Kim MJ, Park SC, Baek JY, Chang HJ, Kim DY, Oh JH (2018) Prediction of lateral pelvic lymph node metastasis in patients with locally advanced rectal cancer with preoperative chemoradiotherapy: focus on MR imaging findings. PLoS ONE 13(4)
30. Matsuda T, Sumi Y, Yamashita K, Hasegawa H, Yamamoto M, Matsuda Y, Kanaji S, Oshikiri T, Nakamura T, Suzuki S, Kakeji Y (2018) Outcomes and prognostic factors of selective lateral pelvic lymph node dissection with preoperative chemoradiotherapy for locally advanced rectal cancer. Int J Colorectal Dis 33(4):367-374

31. Group MS, Shihab OC, Taylor F, Bees N, Blake H, Jeyadevan N, Bleehen R, Blomqvist L, Creagh M, George C, Guthrie A, Massouh H, Peppercorn D, Moran BJ, Heald RJ, Quirke P, Tekkis P, Brown G (2011) Relevance of magnetic resonance imaging-detected pelvic sidewall lymph node involvement in rectal cancer. Br J Surg 98(12):1798-1804

32. Kusters M, Beets GL, van de Velde CJH, Beets-Tan RGH, Marijnen CAM, Rutten HJT, Putter H, Moriya Y (2009) A comparison between the treatment of low rectal cancer in Japan and the Netherlands, focusing on the patterns of local recurrence. Ann Surg 249(2):229-235

33. Kim MJ, Park SC, Kim TH, Kim DY, Kim SY, Baek JY, Chang HJ, Park JW, Oh JH (2016) Is lateral pelvic node dissection necessary after preoperative chemoradiotherapy for rectal cancer patients with initially suspected lateral pelvic node? Surgery 160(2):366-376

34. Hirokazu Nagawa, Tetsuichiro Muto, Koki Sunouchi, Yoshiki Higuchi, Giichiro Tsurita, Toshiaki Watanabe, Sawada T. Randomized, controlled trial of lateral node dissectionvs. nerve-preserving resection in patients with rectal cancer after preoperative radiotherapy. Diseases of the Colon Rectum. 2001;44(9):1274-1280

35. Akasu T, Sugihara K, Moriya Y (2009) Male urinary and sexual functions after mesorectal excision alone or in combination with extended lateral pelvic lymph node dissection for rectal cancer. Ann Surg Oncol 16(10):2779-2786

36. Nagawa H, Muto T, Sunouchi K, Higuchi Y, Tsurita G, Watanabe T, Sawada T (2001) Randomized, controlled trial of lateral node dissection vs nerve-preserving resection in patients with rectal cancer after preoperative radiotherapy. Diseases Colon Rectum 44(9):1274-1280

37. Matsuoka H, Masaki T, Sugiyama M, Atomi Y (2005) Impact of lateral pelvic lymph node dissection on evacuatory and urinary functions following low anterior resection for advanced rectal carcinoma. Langenbecks Arch Surg 390(6):517-522

38. Kennoki Kyo, Shinichi Sameshima, Minoru Takahashi, Taiki Furugori, Sawada T (2006) Impact of autonomic nerve preservation and lateral node dissection on male urogenital function after total mesorectal excision for lower rectal cancer. World J Surg 30(6):1014-1019

39. Fujita S, Akasu T, Mizusawa J, Saito N, Kinugasa Y, Kanemitsu Y, Ohue M, Fujii S, Shiozawa M, Yamaguchi T, Moriya Y, Grp CCS, Grp JCO (2012) Postoperative morbidity and mortality after mesorectal excision with and without lateral lymph node dissection for clinical stage II or stage III lower rectal cancer (JCOG0212): results from a multicentre, randomised controlled, non-inferiority trial. Lancet Oncol 13(6):616-621

40. van Heeswijk MM, Lambregts DM, Palm WM, Hendriks BM, Maas M, Beets GL, Beets-Tan RG (2017) DWI for assessment of rectal cancer nodes after chemoradiotherapy: is the absence of nodes at DWI proof of a negative nodal status? AJR Am J Roentgenol 208(3):W79-W84

41. Engelen SME, Beets-Tan RGH, Lahaye MJ, Lammering G, Jansen RLH, van Dam RM, Konsten J, Leijtens JWA, van de Velde CJH, Beets GL (2010) MRI after chemoradiotherapy of rectal cancer: a useful tool to select patients for local excision. Dis Colon Rectum 53(7):979-986

42. Ishihara S, Kawai K, Tanaka T, Kiyomatsu T, Hata K, Nozawa H, Morikawa T, Watanabe T (2018) Diagnostic value of FDG-PET/ 
CT for lateral pelvic lymph node metastasis in rectal cancer treated with preoperative chemoradiotherapy. Tech Coloproctol

43. Ogura A, Konishi T, Cunningham C, Garcia-Aguilar J, Iversen H, Toda S, Lee IK, Lee HX, Uehara K, Lee P, Putter H, van de Velde CJH, Beets GL, Rutten HJT, Kusters M, Lateral Node Study C. Neoadjuvant (Chemo)radiotherapy With Total Mesorectal Excision Only Is Not Sufficient to Prevent Lateral Local Recurrence in Enlarged Nodes: Results of the Multicenter Lateral Node Study of Patients With Low cT3/4 Rectal Cancer. $J$ Clin Oncol. 2019;37(1):33-43

44. Wei MT, Wu QB, Fan CW, Li Y, Chen XZ, Zhou ZG, Han JH, Wang ZQ. Lateral pelvic lymph node dissection after neoadjuvant chemo-radiation for preoperative enlarged lateral nodes in advanced low rectal cancer: study protocol for a randomized controlled trial. Trials. 2016;17
45. Ogawa S, Hida J, Ike H, Kinugasa T, Ota M, Shinto E, Itabashi M, Kameoka S, Sugihara K (2016) Selection of lymph nodepositive cases based on perirectal and lateral pelvic lymph nodes using magnetic resonance imaging: Study of the Japanese Society for Cancer of the Colon and Rectum. Ann Surg Oncol 23(4):1187-1194

46. Francois Y, Nemoz CJ, Baulieux J, Vignal J, Grandjean JP, Partensky C, Souquet JC, Adeleine P, Gerard JP (1999) Influence of the interval between preoperative radiation therapy and surgery on downstaging and on the rate of sphincter-sparing surgery for rectal cancer: the Lyon R90-01 randomized trial. J Clin Oncol 17(8):2396

Publisher's Note Springer Nature remains neutral with regard to jurisdictional claims in published maps and institutional affiliations. 